\title{
Evaluation and Management of Atopic Dermatitis
}

\author{
J John Kirubakaran ${ }^{1 *}$, Khandavilli Lakshmi Surekha1, Saka Manasa ${ }^{1}$, Amarthi Naga Venkata Chakra \\ Satyanarayana1, KV Srilakshmi ${ }^{1}$, Harinath Palivela², Praveen Kumar ${ }^{3}$, Magharla Dasaratha Dhanaraju ${ }^{4}$
}

\author{
1'Department of Pharmacy Practice, GIET School of Pharmacy, Rajahmundry, Andhra Pradesh, INDIA. \\ ${ }^{2}$ DVL, Dermatologist, Bhavya Clinic, Rajahmundry, Andhra Pradesh, INDIA. \\ ${ }^{3}$ Pediatrist, Vijay Sai Pediatrics Hospital, Rajahmundry, Andhra Pradesh, INDIA. \\ ${ }^{4}$ Principal and Research Director, GIET School of Pharmacy, Rajahmundry, Andhra Pradesh, INDIA.
}

\begin{abstract}
Background: Atopic dermatitis is the most predominant disease seen mostly in children and the prevalence of disease is $10 \%$ to $20 \%$. The pathogenesis of the disease includes immunological, food allergies, pollen grains, breast feeding and secondary infections. Objectives: To identify the cause and to evaluate the appropriate treatment of the patients with atopic dermatitis. Methods: A total of 116 patients are included in the study conducted in skin care and paediatric hospital in the area of Rajahmundry, India. Results: From the study conducted, we observed that the occurrence of the disease is in different age groups and most of the patients are affected by pollen grains $(10 \%)$ followed by pets and respiratory illness $(5.3 \%)$, allergies $(5 \%)$ and pollutants $(4.6 \%)$ and family history $(3.3 \%)$ respectively. On evaluation, the treatment topical steroids are found to be more prominently used followed by emollients, antibiotics, antihistamines and immunomodulators. Conclusion: As per the study, we conclude that the occurrence of the disease is mainly due to pollen grains followed by other determined causes as seen in the area of Rajahmundry.
\end{abstract}

Key words: Atopic dermatitis, Aetiology, Pollen grains, Topical steroids, Emollients.

\section{INTRODUCTION}

Atopic dermatitis (AD), commonly referred as eczema. It is a chronic, relapsing and often intensely pruritic inflammatory disorder of skin allergy. ${ }^{1}$ In infants $\mathrm{AD}$ is often the initial indication of 'atopic march' which leads to asthma, food allergy and allergic rhinitis. ${ }^{2}$ It is the most common childhood disorder with a prevalence of $10 \%$ to $20 \%$ which is increased up to two to three-fold since the 1970 's. ${ }^{3}$

$\mathrm{AD}$ is the chronic inflammatory disease which is mainly caused due to triggering due to imbalance of T-helper cells, ${ }^{4}$ pollen grains mainly causes hypersensitivity reaction due to aeroallergens of Dactylisglomerata or pyrethrum plants, ${ }^{5,6}$ foods like milk, eggs, peanuts etc. can provoke allergies, ${ }^{7}$ breastfeeding on the mother with any known respiratory illness causes atopic dermatitis in children ${ }^{8}$ and secondary infections by bacteria (for eg. $S$. aureus) leads to lesions by altering the sebum and sweat secretion., ${ }^{9,10}$

The American Academy of Dermatology has suggested some universal diagnostic criteria for atopic dermatitis which includes essential features like pruritus, atopy, eczematous changes that are acute, sub-acute and chronic. ${ }^{11}$ Clinically, the morphology and distribution of eczema in AD patients varied based on the age of infants (birth to below 2 years of age) typically present with erythematous papules and vesicles on the cheeks, forehead and scalp, whereas children (above 2 years of age) exhibit dry skin and lichenified papules and plaques in flexural areas of the limbs. In adulthood, the predominant areas of eczema are the flexural folds, the face and neck, the upper arms and back, hands, feet, fingers and toes. ${ }^{2}$

Skin-directed therapies should be the first approach to the management. AD results from an abnormality of the skin barriers which includes drying of the skin due to filaggrin deficiency and reduce natural skin lipids. Emollients ${ }^{4,12}$ are used to repair and maintain healthy skin barriers by retaining
DOI: 10.5530/ijopp.13.2.22

Address for correspondence: Mr. J. John Kirubakaran Associate Professor, Department of Pharmacy Practice, GIET School of Pharmacy, Rajahmundry, Andhra Pradesh, INDIA.

Phone no: +917893277176 Email Id: john.mpharm@gmail. com

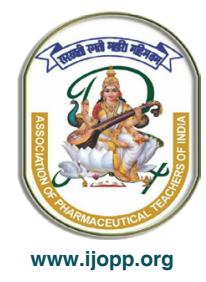


and redistributing water content. Topical steroids ${ }^{4,13}$ are mainly used to treat moderate to severe atopic dermatitis and beneficial for suppressing inflammatory responses. The itching caused due to atopic dermatitis is treated by giving sedative anti-histamines ${ }^{3,4}$ and to secondary infections antibiotics ${ }^{14}$ are used. In severity of atopic dermatitis immunomodulators ${ }^{15,16}$ are used.

\section{METHODOLOGY}

Patients from a skin care and paediatric hospital with the typical clinical features of atopic dermatitis from the outpatient department were included in the study. Patients who were diagnosed with atopic dermatitis by the dermatologist in the skin care clinic and the physician from the paediatric hospital are included in the study and those with other skin diseases are excluded. The study was approved by the institutional ethical committee.

The study was conducted for 6 months and collected the patient data with the case sheets and questionnaire. The questionnaire is prepared based on prospect of the disease in different kinds of literature.

Totally 116 cases of the disease were collected from both the hospitals. The cases are collected with the cooperation of the parents of the children.

The main objective of the study is to identify the aetiology of the disease and to determine the appropriate treatment for the patients with atopic dermatitis.

\section{RESULTS}

According to the study, a total of 116 patients from both rural and urban area attending primary dermatology care clinic and children speciality hospitals were interviewed in the study. Among them the majority were female who constituting of $58.26 \%(n=67)$ and males constituting of $41.74 \%(n=48)$ (Figure 1).

According to the study, the age distribution 1-3 years constituting $26.96 \%(n=31)$ were more affected, followed by $4-6$ months constituting $21.74 \%(n=25)$ and $4-6$ years constituting $13.91 \%(n=16)$ followed by rest of the age groups. Here in the following table are cited by age group distribution relatively presenting to the dermatology clinic and the children speciality hospital. (Figure 2)

As per the study, there are many etiological factors causing atopic dermatitis. Pollen grains from the pyrethrum plants constitutes the major source of cause for the disease. (Figure 3)
We also observed results of following types of atopic dermatitis

\section{Seborrheic eczema}

a) Seborrheic dermatitis of infancy of abdomen (Figure 4)

b) Adult type seborhheic dermatitis (Figure 5)

\section{Contact dermatitis}

\section{Discoid dermatitis (Figure 6) \\ Lichenoid dermatitis (Figure 7)}

\section{Statistical Data Analysis}

As per our study, the severity of disease is mainly seen in the paediatric population. Hence this population are categorized into specific groups accordingly,1-3 months, 4-6months, 7-11 months,1-3years,4-6years,79 years and 10 years and above. Based on these age groups the cause of the disease is determined. To calculate these data ANOVA (analysis of variance) is utilized. ANOVA is mainly used to compare multiple means of different groups.

ANOVA is used to determine whether there are any statistically significant differences between the means of three or more independent (unrelated) groups. To apply this test, we have taken the age groups based on their age i.e., below one year and above one year. The causes of the disease listed are as follows- pollen, pets, pollutants (industrial), allergies, asthma (i.e. if any respiratory illness), family history (if any).

The data for below one-year age groups is calculated by using ANOVA. The $p$ value is to be determined to know the significant means. The $\mathrm{f}$ value obtained from the ANOVA table is 5.57 and the $p$ value for this

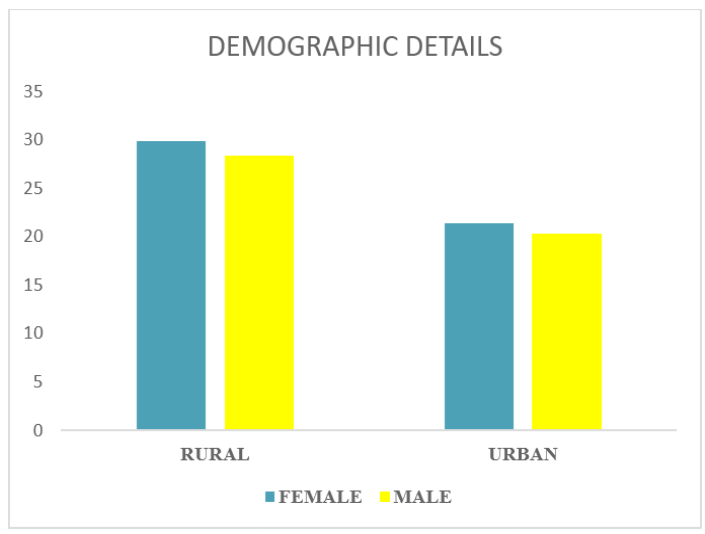

Figure 1: Demography of the study population. 
distribution is 4.34 , which is lower than the obtained $\mathrm{f}$ value. Hence the hypothesis is significant. The means of the distribution is also calculated. There is no significant difference in the average number of affected people due to the sources (causes) in different age groups ranging from 1-3 months, 4-6 months, 7-11 months respectively. The means are arranged according to their distribution. The highest average number of affected areas or age groups due to the sources are 4-6 months (7.1\%), followed by $1-3$ months (5.3\%) and least in 7-11 months $(4.8 \%)$.

Also the average number of affected people due to different sources are equal, ranging $10 \%$ due to pollen, $5.3 \%$ due to pets and respiratory illness, $5 \%$ due to allergies, $4.6 \%$ due to pollutants and least $3.3 \%$ due to family history.

Hence the sources of causes are highly significant in age groups i.e. 4-6 months. The average number of persons affected due to pollen $(10 \%)$ is highly significant over the other sources. The means of other sources are at par ranging from 3.3 to 5.3 .

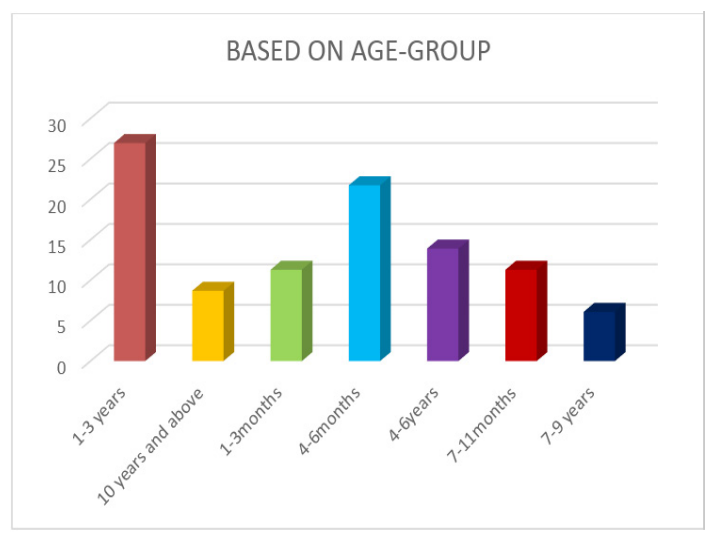

Figure 2: Age group distribution in the study population.

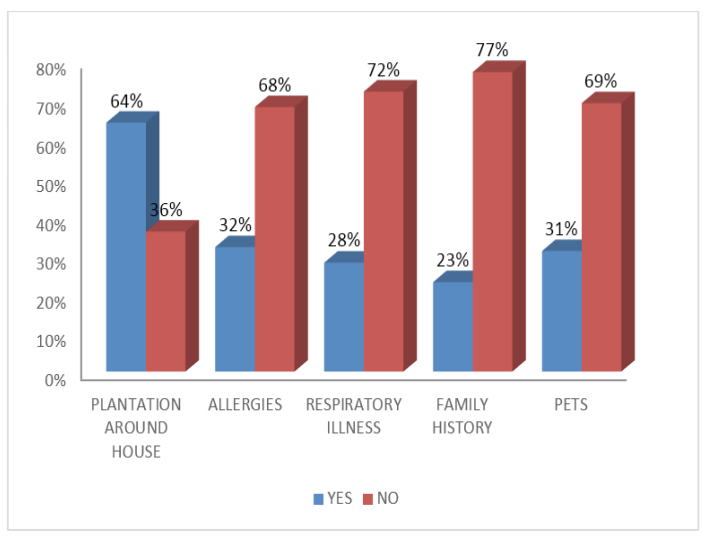

Figure 3: Etiological Factors in the Patients.
The data for above one-year age groups is calculated as the previous one and means of the data are determined. The $f$ value from the ANOVA table is found to be 0.39 which is lower than the $\mathrm{p}$ value (4.56). There is no significant difference in the means of average number of affected people due to the sources in different age groups ranging from 1-3 years, 4-6 years, 7-9 years and 10 years and above. The average number of persons affected due to allergies in different age groups are equal with the highest i.e. 10\% in age group $1-3$ years followed by $6.3 \%$ in $4-6$ years, $3.8 \%$ in 10 years and above and lowest i.e. $3.3 \%$ in age group $7-9$ years.

The average number of persons affected due to pollen are $10.75 \%$, pets and allergies are $5.5 \%$, family history is $4.5 \%$ followed by pollutants and respiratory illness i.e. $4.25 \%$. Hence the sources of causes are highly significant in 1-3 years' age group. The average number of persons affected due to pollen are $10.75 \%$ is highly significant over the other sources. The persons affected by other sources are at par, ranging from 4.25 to $5.5 \%$.

Hence from both the statistical data, we observed that most of the people are affected majorly by the pollen

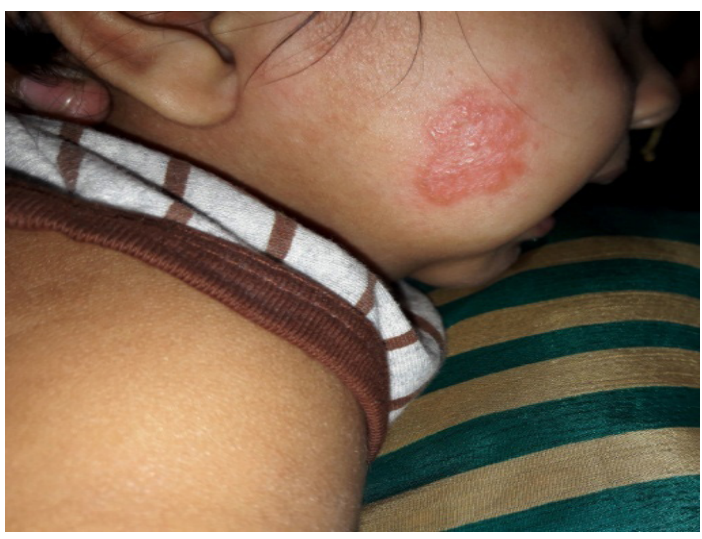

Figure 4: a. Seborrheic eczema on cheek.

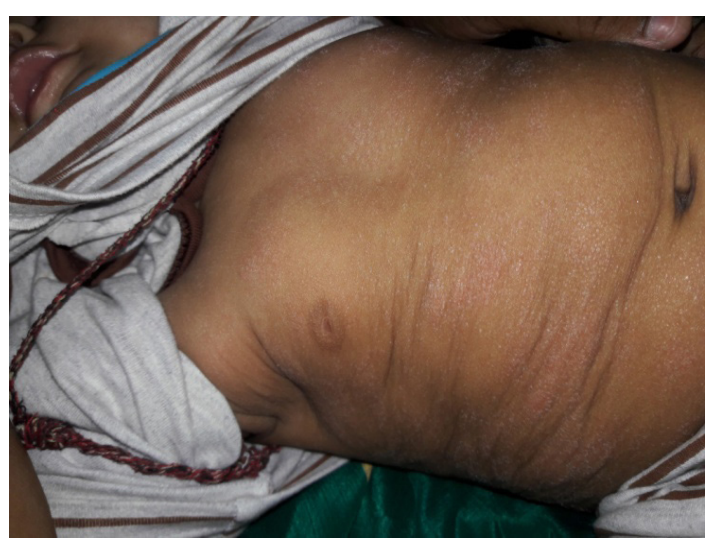

Figure 4: b. Seborrheic dermatitis of infancy of abdomen. 
grains from the pyrethrum plants $(n=73)$ followed by pets and respiratory illness $(n=38)$, pollutants and allergies $(n=34)$ and family history $(n=28)$ respectively. It also gives the distribution of the age groups which are mostly affected. These are arranged in the order from most prone groups to less prone i.e. 1-3 years $(n=60)$, 4-6 months ( $n=43), 4-6$ years $(n=38)$, followed by $1-3$ months $(n=32)$, 7-11 months $(n=29)$ and the least in 6-10 years and 10 years and above $(n=20)$.

The evaluation of the treatment is also determined by ANOVA. In this data, the numerical value for 0 is transformed by using the formula $V_{X}+1$. This transformed value is obtained and the ANOVA is done for the data. The $\mathrm{f}$ value obtained is 9 which is greater than the $p$ value (4.77).

From the ANOVA table, the treatments are significantly different with respect to its prescription. The highest is topical steroids (20\%) followed by emollients $(19 \%)$, antibiotics ( $8 \%$, anti-histamines $(7 \%)$ and the least is immunomodulators $(1.4 \%)$. (Table 1$)$

Regarding the average prescription for age groups, they

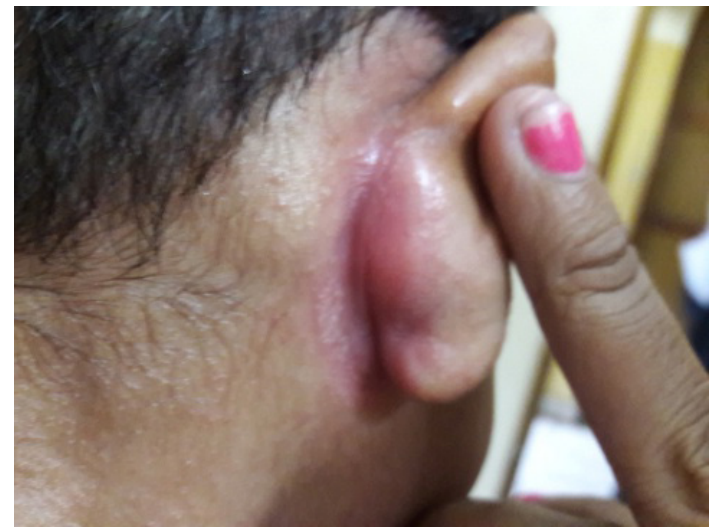

Figure 5: Adult type seborrheic dermatitis of ear (atopic dermatitis).

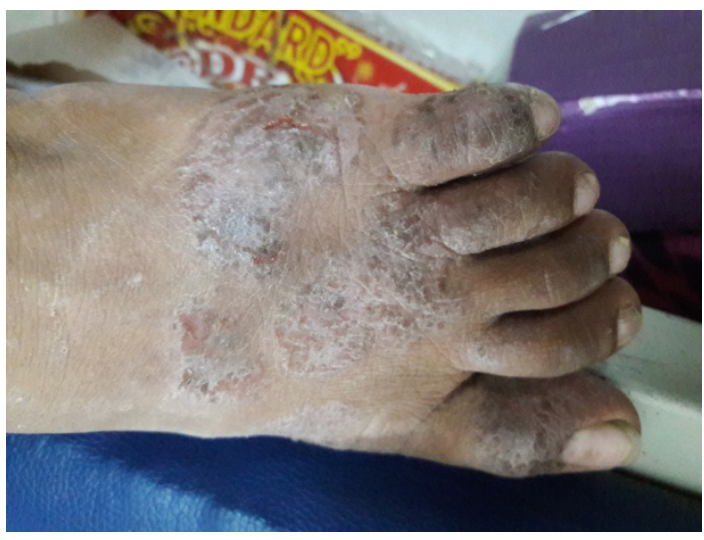

Figure 6: a. Discoid eczema of foot. are also significantly different, the highest number of prescriptions for below one-year age groups is higher i.e. $22.2 \%$ followed by $1-3$ years $(16 \%)$, $4-6$ years $(9 \%)$, $>10$ years $(5.2 \%)$ and the lower is $6-10$ years $(2.8 \%)$. It is interesting to know that the average prescription in all the three age groups are at par. These three age groups are significantly higher prescription over the age groups 6-10 years and $>10$ years. Interestingly both these groups are at par with regard to average prescription.

\section{DISCUSSION}

Based on the study conducted by Simon Francis Thomsen, ${ }^{4}$ atopic dermatitis affects about one-fifth of all individuals but the prevalence increased substantially throughout the world in so called industrialised countries. It is mostly seen in many countries including United States and Australia. According to Simon Francis Thomsen, atopic dermatitis is mostly seen in children under age of 12 years. In our study, children below 10 years of age are mostly affected by the atopic dermatitis similar to the study done by Simon Francis Thomsen.

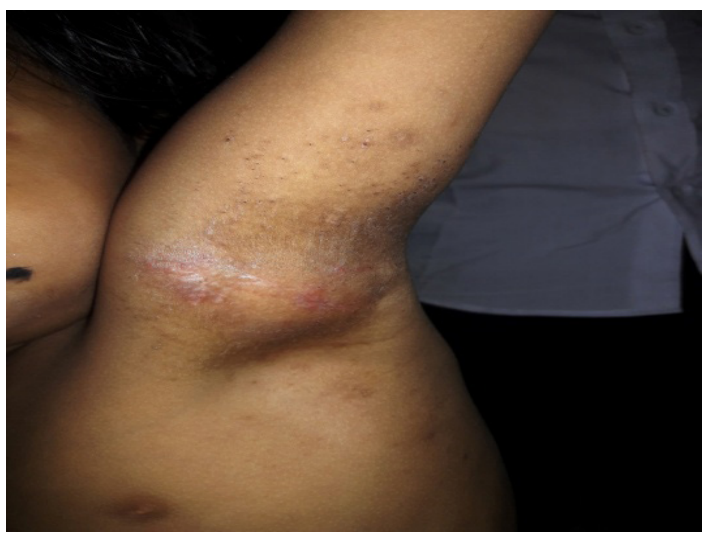

Figure 6: b. Discoid eczema with secondary infection of $S$. aureus.

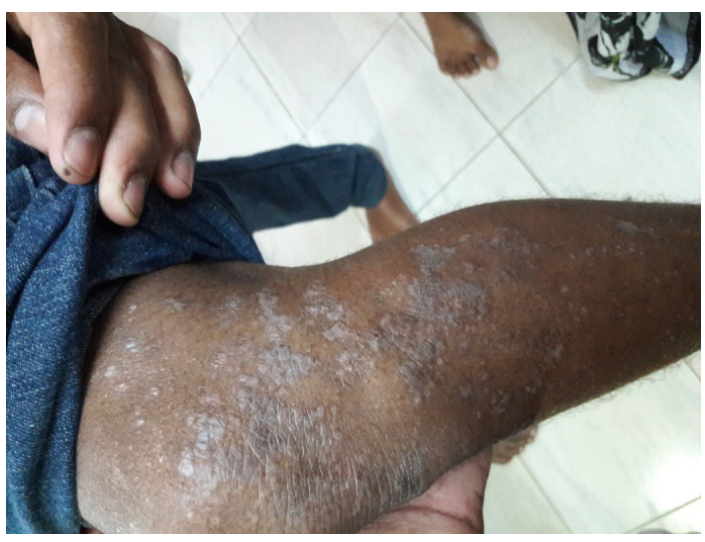

Figure 7: Lichenoid dermatitis of knee. 


\begin{tabular}{cccccc}
\hline \multicolumn{6}{c}{ Table 1: List of Drugs used in the study population. } \\
\hline Treatment & $<1$ Year & $\begin{array}{c}\mathbf{1 - 3} \\
\text { Year }\end{array}$ & $\begin{array}{c}\mathbf{4 - 6} \\
\text { Year }\end{array}$ & $\begin{array}{c}\mathbf{7 - 9} \\
\text { Year }\end{array}$ & $\begin{array}{c}>10 \\
\text { Year }\end{array}$ \\
\hline Emollients & 44 & 28 & 13 & 4 & 8 \\
Topical steroids & 48 & 23 & 12 & 5 & 9 \\
Immunomodulators & 0 & 2 & 3 & 2 & 0 \\
Anti-histamines & 8 & 15 & 10 & 0 & 0 \\
Antibiotics & 11 & 12 & 7 & 3 & 7 \\
\hline
\end{tabular}

According to the study conducted by the author Küster and Petersen M, the children mostly affected by the disease are females than males, in our study too we found that females $(58 \%)$ were mostly affected than males $(42 \%)$. As given by the author Simon Francis Thomsen, the children affected are below 12 years. Similarly, in our study we obtained 116 cases in which children below 10 years are mostly affected. The order of age groups affected is depicted as 51\% (1-3 years), 37\% (4-6 months), $33 \%$ (4-6 years) followed by the remaining groups.

By the study of Lastl Allison J. Burbank et al. it is proven that the major cause of the disease is by the triggering factors. Here in our study, we listed some of the triggering factors based on the literatures. The percentages of these sources are calculated and are obtained as, pollen $(63 \%)$ followed by pets and respiratory illness $(38 \%)$, pollutants and allergies (34\%) and family history (28\%). We observed that pollen is the highest chance of getting the atopic dermatitis in our study which is similar to the study conducted by Lastl Allison J. Burbank et al. Similarly, the study conducted by the author Simon Francis Thomsen depicts the environmental factors, family history, any respiratory illnesses etc. mainly contribute for the cause of the atopic dermatitis. Here in our study, we obtained similar factors as sources.

In our study, we observed that rural population $(51 \%)$ is mostly affected than the urban population (49\%) as in the conducted by Simon Francis Thomsen. The extremities which are mainly affected are face, scalp in infants and on elbows, knees, abdomen in older children.

As per our study, we observed various types of atopic dermatitis. These mainly include seborrheic eczema, seborrheic dermatitis, lichenoid dermatitis, discoid eczema and lichenified eczema etc. which is similar to the study done by ${ }^{6}$ Hywel C. Williams from England journal of medicine.

Coming to the treatment of the atopic dermatitis, topical corticosteroids and emollients are the mostly prescribed drugs for the relief of the symptoms.${ }^{17}$ Other treatments include antibiotics given during the presence of any bacterial infection, anti-histamines and topical calcineurin inhibitors. These treatments are given appropriately for the prevention of the disease. The study conducted by the authors Hywel C. Williams and Simon Francis Thomsen depicts the similarity of the treatment we obtained in our study.

Comparing our results with the studies of Lastl Allison J. Burbank et al. Hywel C. Williams and Simon Francis Thomsen, we did not find any differences regarding the aetiology and treatment of the atopic dermatitis. So as per our study pattern, the causes of the atopic dermatitis are similar in population throughout the world.

\section{CONCLUSION}

The study conducted depicts the conclusion which is similar to many other studies conducted worldwide by many authors. Atopic dermatitis is mostly prevalent in many industrialised countries. The prevalence of $\mathrm{AD}$ is estimated to be $15-20 \%$ in children and $1-3 \%$ in adults. It seems to be increased to two to three fold. Based on the studies that are conducted it is shown that the incidence is increased to $20 \%$ in children throughout the world. In India the prevalence ranged from $0.9 \%$ than the previous decades. It is mainly caused due to various triggering factors. Hence the prevalence and incidence can be decreased if the reduction in these triggering factors is achieved.

During the study process conducted, a total of 116 patients identified and diagnosed with the atopic dermatitis. As the cause of the atopic dermatitis is unknown several triggering factors are taken into the consideration like plantation (pollen grains, pets, pollutants, allergies (if any), any previous respiratory illness (asthma, rhinitis), family history (if any) and others include industrialised areas. Hence a questionnaire is prepared based on the presence of these triggering factors and the study is done. The age groups which are mostly affected are below 10 years mostly and very little in adults. In the age groups below 10 years, the mostly affected age groups are $1-3$ years (52\%), 4-6 months (37\%) followed by the other age groups. By taking gender into consideration, females $(68 \%)$ are mostly affected than males $(42 \%)$. Coming to the causes of the disease the majority cases are of plantation i.e. pollen (63\%) followed by pets and respiratory illness (38\%), pollutants and allergies (34\%) and family history $(28 \%)$.

The onset of the atopic dermatitis is mainly seen during the winter monsoon as the allergens are mostly triggered 
during this season. The disease mostly consists of itchy, scaly rashes which are seen all over the body mostly on the areas of face i.e. cheeks, crown of the head and abdomen followed by both upper and lower limbs. In some cases, discharge of blood from the rashes is also seen due to increases itching.

Topical steroids and emollients are mostly used for the symptomatic relief of atopic dermatitis. In severe cases immune modulators are prescribed for the atopic dermatitis. Antibiotics are also prescribed where in the cause of the disease is mainly due to any bacterial organism and to reduce the infection.

As atopic dermatitis mainly caused due to dryness of the skin, moisturizers are to be applied daily to retain the moisture to the skin.

\section{ACKNOWLEDGEMENT}

We are very thankful to Bhavya Hospital and Vijay Sai Paediatric Clinic, Rajahmundry for providing us the necessary facilities and amenities to carry out our project work effectively and efficiently.

I take the privilege to thank each and every patient for their valuable cooperation for sparing their time in filling the questionnaire forms.

\section{CONFLICT OF INTEREST}

There are no conflicts of interest

\section{SUMMARY}

Topical steroids and emollients are mostly used for the symptomatic relief of atopic dermatitis. In severe cases immune modulators are prescribed for the atopic dermatitis.

As atopic dermatitis mainly caused due to dryness of the skin, moisturizers are to be applied daily to retain the moisture to the skin.

\section{REFERENCES}

1. Tollefson MM, Bruckner AL. Section on Dermatology SO Atopic dermatitis: Skindirected management. Pediatrics. 2014;134(6):e1735-44. Available from: http:// www.ncbi.nlm.nih.gov/pubmed/25422009

2. Egawa G, Weninger W. Pathogenesis of atopic dermatitis: A short review. Cogent Biol. 2015;1(1):1. Available from: https://www.cogentoa.com/ article/10.1080/23312025.2015.1103459

3. Botskariova SKA. Atopic Dermatitis: Insights on Pathogenesis, Evaluation and Management. J Allergy Ther. 2014;05(06):1. Available from: https://www. omicsonline.org/open-access/atopic-dermatitis-insights-on-pathogenesisevaluation-and-management-2155-6121.1000195.php?aid=33762

4. Lee JH, Son SW, Cho SH. A Comprehensive Review of the Treatment of Atopic Eczema. Allergy Asthma Immunol Res. 2016;8(3):181-90. Available from: http:// www.ncbi.nlm.nih.gov/pubmed/26922927

5. Thomsen SF. Atopic dermatitis: Natural history, diagnosis and treatment. ISRN Allergy. 2014;2014(354250):1-7. Available from: http://www.ncbi.nlm.nih.gov/ pubmed/25006501

6. Williams HC. Atopic Dermatitis. N Engl J Med. 2005;352(22):2314-24. Available from: http://www.nejm.org/doi/abs/10.1056/NEJMcp042803

7. Eyerich K, Novak N. Immunology of atopic eczema: Overcoming the Th1/ Th2 paradigm. Allergy. 2013;68(8):974-82. Available from: http://doi.wiley. com/10.1111/all.12184

8. Gong JQ, Lin L, Lin T, Hao F, Zeng FQ, Bi ZG, et al. Skin colonization by Staphylococcus aureus in patients with eczema and atopic dermatitis and relevant combined topical therapy: A double-blind multicentre randomized controlled trial. Br J Dermatol. 2006;155(4):680-7. Available from: http://www. ncbi.nlm.nih.gov/pubmed/16965415

9. Leyden JJ, Marples RR, Kligman AM. Staphylococcus aureus in the lesions of atopic dermatitis. Br J Dermatol. 1974;90(5):525-30. Available from: http://www. ncbi.nlm.nih.gov/pubmed/4601016

10. Gloor M, Peters GSD. On the resident aerobic bacterial skin flora in unaffected skin of patients with atopic dermatitis and in healthy controls. Dermatologica. 1982;164(4):258-65. Available from: https://www.unboundmedicine.com/ medline/citation/7044853/On_the_resident_aerobic_bacterial_skin_flora_in_ unaffected_skin_of_patients_with_atopic_dermatitis_and_in_healthy_controls_

11. Roth RR, James WD. Microbiology of the skin: Resident flora, ecology, infection. J Am Acad Dermatol. 1989;20(3):367-90. Available from: http://www.ncbi.nlm. nih.gov/pubmed/2645319

12. Savolainen J, Lammintausta K, Kalimo K, Viander M. Candida albicans and atopic dermatitis. Clin Exp Allergy. 1993;23(4):332-9. Available from: http:// www.ncbi.nlm.nih.gov/pubmed/8319131

13. Wilson BB, Deuell B, Mills TA. Atopic dermatitis associated with dermatophyte infection and Trichophyton hypersensitivity. Cutis. 1993;51(3):191-2. Available from: http://www.ncbi.nlm.nih.gov/pubmed/8444052

14. Rajka G. History and Nomenclature. In: Essential Aspects of Atopic Dermatitis. Berlin, Heidelberg: Springer Berlin Heidelberg. 1989;1-3. Available from: http:// www.springerlink.com/index/10.1007/978-3-642-74766-3_1

15. Eyerich K, Huss-Marp J, Darsow U, Wollenberg A, Foerster S, Ring J, et al. Pollen Grains Induce a Rapid and Biphasic Eczematous Immune Response in Atopic Eczema Patients. Int Arch Allergy Immunol. 2008;145(3):213-23. Available from: http://www.ncbi.nlm.nih.gov/pubmed/17914273

16. Werfel T, Heratizadeh A, Niebuhr M, Kapp A, Roesner LM, Karch A, et al. Exacerbation of atopic dermatitis on grass pollen exposure in an environmental challenge chamber. J Allergy Clin Immunol. 2015;136(1):96-103. Available from: http://www.ncbi.nlm.nih.gov/pubmed/26044854

17. Saeki H, Furue M, Furukawa F, Hide M, Ohtsuki M, Katayama I, et al. Guidelines for management of atopic dermatitis. J Dermatol. 2009;36(10):563-77. Available from: http://doi.wiley.com/10.1111/j.1346-8138.2009.00706.x 